DOI: 10.3901/JME.2019.14.132

\title{
低地板有轨电车防折弯系统及动力学仿真
}

\author{
王小超 陆正刚 \\ (同济大学铁道与城市轨道交通研究院 上海 201804)
}

\begin{abstract}
摘要: 针对低地板有轨电车及引进的防折弯液压系统, 分析防折弯系统中相关重要参数对液压系统防折弯功能和二系减振功 能的影响。提出防折弯液压系统与低地板有轨电车动力学模型联合仿真的方法, 研究防折弯液压系统对低地板有轨电车通过 曲线性能的影响。液压系统参数研究表明, 主阀块旁通节流孔直径和缓冲油缸弹簧刚度主要影响液压系统的防折弯功能, 缓 冲阀块阻尼孔直径和限压阀开启压力主要影响液压系统的二系减振功能。联合仿真结果显示, 防折弯系统能够很好地保持有 轨电车前后车体与转向架之间相对回转的一致性, 抑制车体过大的横向位移和前后车体间的相对位移。建立的抗折弯液压系 统-列车动力学联合仿真模型, 可用于研究抗折弯液压系统对低地板有轨电车通过曲线时列车各项性能的影响。
\end{abstract}

关键词: 有轨电车; 防折弯系统; 参数研究; 机械-液压联合仿真

中图分类号: U270

\section{Anti-kink System and Dynamics Simulation of Low Floor Tram}

\author{
WANG Xiaochao LU Zhenggang
}

(Institute of Rail Transit, Tongji University, Shanghai 201804)

\begin{abstract}
Based on the low floor tram and the anti-kink hydraulic system introduced, the impact of important parameters in the anti-kink system on the anti-kink function and secondary damping functions of the hydraulic system is analyzed. A method of co-simulation of anti-kink hydraulic system and low-floor tram dynamic model is proposed, and the influence of anti-kink hydraulic system on the curve performance of low-floor trams is studied. The results show that the diameter of bypass orifice of main valve block and the spring stiffness of buffer cylinder mainly affect the anti-kink function of hydraulic system. The diameter of damping orifice of orifice block and the opening pressure of pressure-limiting valve mainly affect the damping function of hydraulic system. The co-simulation results show that the anti-kink system can well maintain the relative kink between vehicles, and inhibit the excessive lateral displacement and the relative displacement between the front and rear bodies. The co-simulation of anti-kink hydraulic system and tram dynamics model can be used to study the impact of the anti-kink hydraulic system on the performance of the train when the low-floor tram passes the curves.
\end{abstract}

Key words: modern tram; anti-kink system; parameter research; co-simulation between mechanical and hydraulic

\section{0 前言}

储能式低地板有轨电车采用模块化设计、超级 电容供电 ${ }^{[1-3]} 、 100 \%$ 低地板 ${ }^{[4-5]}$ 、防折弯系统等多项 先进技术。四模块低地板有轨电车由三节动车 $M c 1$ 、 $\mathrm{M} 、 \mathrm{Mc} 2$ 车以及一节拖车 $\mathrm{T}$ 车组成 ${ }^{[6]}$, 每节车辆下 装配一个转向架, $\mathrm{Mc} 1$ 车与 $\mathrm{T}$ 车、 $\mathrm{M}$ 车与 $\mathrm{Mc} 2$ 车 之间通过单铰接连接, 只允许两车之间绕铰接中心 在水平面内转动。 $\mathrm{T}$ 车和 $\mathrm{M}$ 车之间采用双铰连接, 对各自由度没有限制(图 1)。由于该种有轨电车采用 每节车厢下一个转向架的结构, 因此在运行过程中,

20180906 收到初稿, 20190325 收到修改稿
车体相对于转向架运动趋势较大。在牵引模块或制 动模块出现故障的情况下, 存在运动趋势的不确定 性, 因此对其进行曲线通过性能及运行平稳性研究 都十分必要。张徐 ${ }^{[7]}$ 建立了四模块 100\%低地板有轨 电车模型, 并分析了列车在不同曲线线路上的通过 性能。李创等 ${ }^{[8]}$ 系统地分析了独立车轮转向架车辆 曲线通过性能, 着重对独立车轮和传统轮对的磨耗 状况进行了比较。CZAUDERNA 等 ${ }^{[9]}$ 对低地板车间 四连杆车钩系统进行弹性运动分析, 试验研究了车 钩上金属杆与弹性祄套连接处的刚度特性。

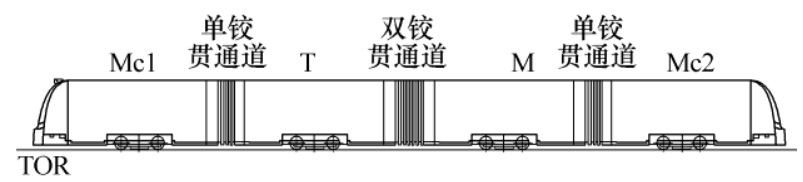

图 1 低地板有轨电车总体结构 
防折弯液压系统是一种低地板车辅助导向装置, 其液压原理图如图 2 所示, $\mathrm{Mc} 1$ 车和 $\mathrm{T}$ 车、 $\mathrm{M}$ 车和 Mc2 车各有一套独立的液压系统, 两套液压系统共用 一套控制器 BK3, 液压系统主要由蓄能器、主阀块组、 锁紧阀块组、缓冲阀块组、缓冲油缸、转向油缸组成, BK3 接受车辆运行状态指令对液压系统进行控制。文 献[10-12]对防折弯液压系统的总体结构和工作机理 进行了概述, 黄江伟等 ${ }^{[13]}$ 对防折弯液压系统进行了结
构静强度试验及列车曲线通过试验。目前对防折弯系 统的研究大多局限于工作原理分析, 缺少对整套系统 的理论研究和液压系统中各部件相关参数分析, 通过 试验研究虽然能够真实反应该套系统的作用机理, 但 由于试验成本以及试验系统的复杂性不能对该系统 各个部件参数进行详尽试验。此时, 对防折弯液压系 统进行理论分析, 建立防折弯液压系统与低地板车动 力学系统联合仿真模型则显得尤为必要。

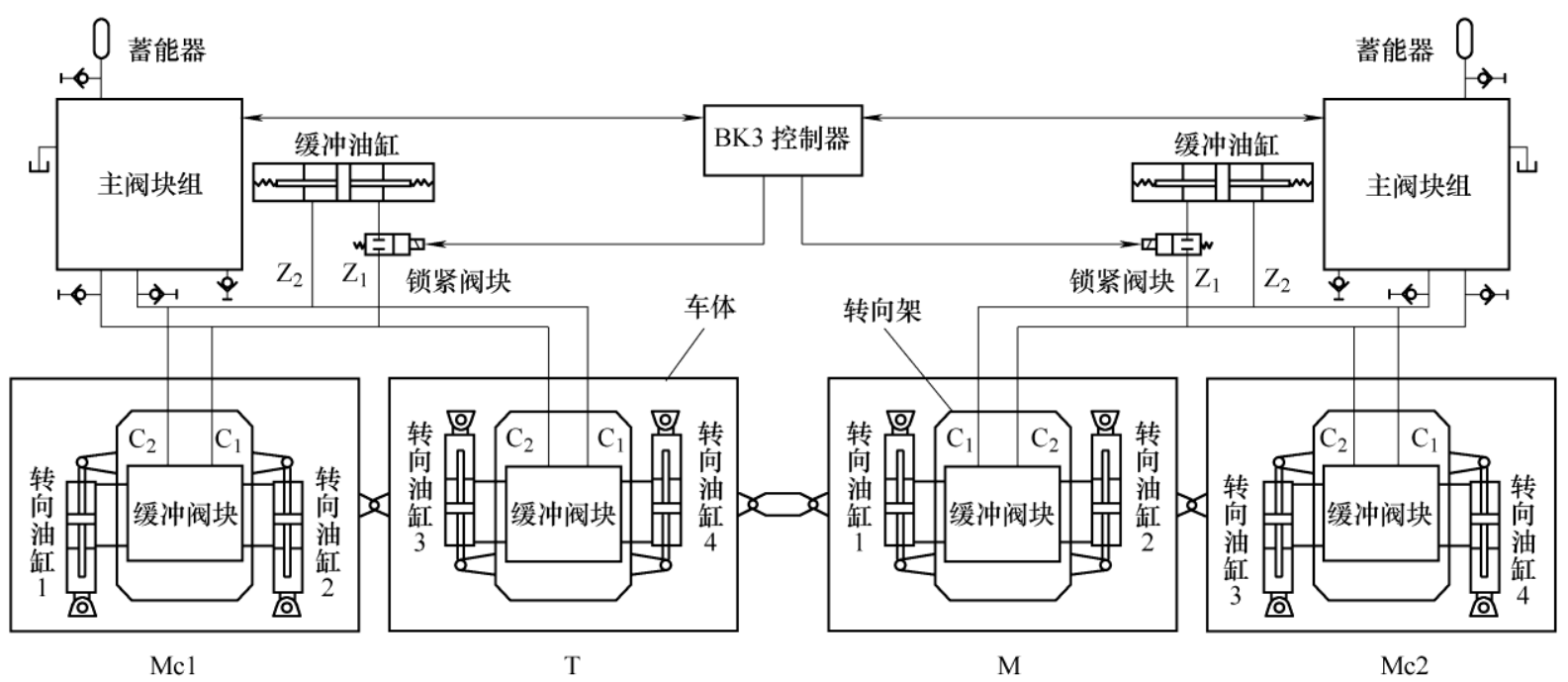

图 2 防折弯系统液压原理图

本文分析了防折弯液压系统对列车运行过程主 要产生两种运动约束功能, 一是车与车之间的防折 弯功能, 二是车体与转向架之间的二系减振功能。 针对两种约束功能分别建立液压系统防折弯功能数 学模型和二系减振功能数学模型, 并对每种约束功 能产生重要影响的液压系统参数进行参数分析。最 后建立了列车动力学-防折弯液压系统联合仿真模 型, 对比分析了在有、无防折弯系统对列车动力学 性能的影响。

\section{1 防折弯系统数学模型}

防折弯系统通过增加低地板车辆的运动约束来 防止单个车体模块行驶于直线和曲线轨道上以及驶 入和驶出曲线时发生不必要的位移和运动 ${ }^{[13]}$ 。而该 系统对行驶车辆的约束主要分为两个方面(图 3), 一 是前后车体间的约束, 二是车体与转向架之间的约 束。前后车体间的约束能够使得同一液压模块连接 的两节车相对于各自转向架绕 $Z$ 轴的转角一致, 从 而实现车体之间的防折弯功能; 车体与转向架之间 的约束能够抑制同一节车中车体相对于转向架横摆 和摇头过程中的振动, 从而实现车体与转向架之间 的二系减振功能。针对防折弯液压系统对列车的两
种约束功能, 分别建立液压系统防折弯功能数学模 型和二系减振功能数学模型。

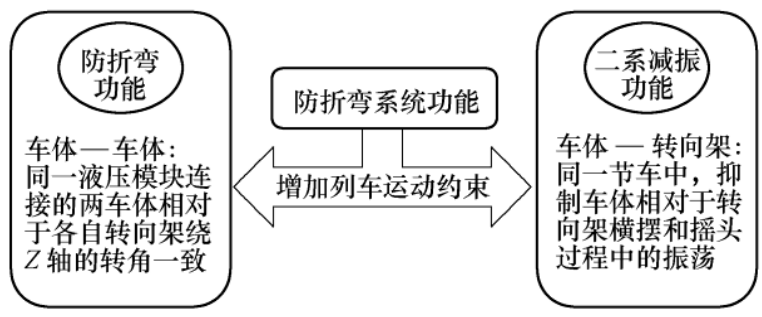

图 3 防折弯系统功能分析

\section{1 防折弯功能数学模型}

防折弯系统可使一个单元内的两个车体模块相 对于各自转向架的摇头角 $\alpha_{1}$ 和 $\alpha_{2}$ 保持相等。当 $\alpha_{1}$ 与 $\alpha_{2}$ 不相等的时候, 防折弯系统会在转向架与车体 间产生一个反向的扭矩 $M_{\alpha}$, 使得 $\alpha_{1}, \alpha_{2}$ 倾向于一 致。一般假设这个扭矩与摇头角之间存在这样一个 关系，即

$$
M_{\alpha 1}=-M_{\alpha 2}=K_{\alpha}\left(\alpha_{1}-\alpha_{2}\right)
$$

式中, $M_{\alpha 1}$ 和 $M_{\alpha 2}$ 为前后两个车体模块的转向架与 车体间的扭矩; $K_{\alpha}$ 为车体间辅助回转刚度。

液压缸的两端分别连接车体与转向架, 则车体 与转向架之间的相对转角 $\alpha$ 与液压缸运动部件的位 移 $Y$ 变化关系式为 


$$
Y=L \alpha
$$

式中, $L$ 为防折弯系统与车体连接点距离转向架中 心纵向距离。

当车体与转向架之间仅产生相对摇头运动时, 同一个模块内的两个控制液压缸位移和输出流量总 是保持相同，因此将同一模块内的两个控制液压缸 合并为一个控制液压缸, 位移为 $Y$, 作用力为 $F$, $F$ 与 $M_{\alpha}$ 之间有如下关系

$$
M_{\alpha}=2 L F
$$

假设单个液压缸活塞面积和初始容积为 $A_{0}$ 和 $V_{0}$, 则合并后的控制液压缸活塞面积和初始容积是 $2 A_{0}$ 和 $2 V_{0}$ 。在分析回转刚度 $K_{\alpha}$ 的时候, 只考虑控 制油缸活塞杆位移变化对系统输出扭矩的影响, 不 考虑速度变化的影响。因此在分析车体间辅助回转 刚度 $K_{\alpha}$ 的影响因素时可忽略缓冲阀组的作用。此时 液压系统防折弯功能模型可简化如图 4 所示。

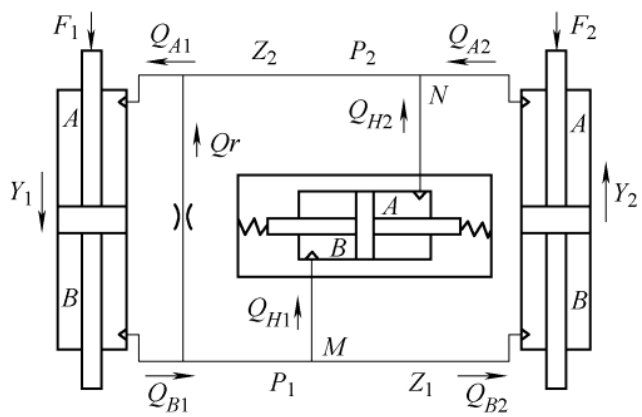

图 4 液压系统防折弯功能简化模型

假设控制液压缸 1 在 $F_{1}$ 作用下产生向下的位移 $Y_{1}$, 控制液压缸 2 在压力作用下有向上的位移 $Y_{2}$, 阻力为 $F_{2}$, 如图 3 所示。

控制液压缸 1 处, 根据流量连续性公式有 ${ }^{[14]}$

$$
\begin{aligned}
& 2 A_{0} \frac{\mathrm{d} Y_{1}}{\mathrm{~d} t}-Q_{B 1}=\frac{2 V_{0}-2 A_{0} Y_{1}}{\beta_{e}} \frac{\mathrm{d} P_{1}}{\mathrm{~d} t} \\
& Q_{A 1}-2 A_{0} \frac{\mathrm{d} Y_{1}}{\mathrm{~d} t}=\frac{2 V_{0}+2 A_{0} Y_{1}}{\beta_{e}} \frac{\mathrm{d} p_{2}}{\mathrm{~d} t}
\end{aligned}
$$

式中, $Q_{B 1}$ 为 $B$ 腔出油流量; $Q_{A 1}$ 为 $A$ 腔进油流量; $\beta_{e}$ 为油液的体积模量。

单个控制液压缸运动部件运动微分方程如下

$$
m_{k} \frac{\mathrm{d}^{2} Y_{1}}{\mathrm{~d} t^{2}}=F_{1}-\left(P_{1}-P_{2}\right) A_{0}-B \frac{\mathrm{d} Y_{1}}{\mathrm{~d} t}
$$

式中, $m_{k}$ 为控制液压缸运动部件质量; $B$ 为液压缸 活塞运动阻尼系数。

同理, 缓冲液压缸 $A$ 腔与 $B$ 腔流量连续性方程

$$
\begin{aligned}
& A_{H} \frac{\mathrm{d} x}{\mathrm{~d} t}-Q_{H 2}=\frac{V_{1}-A_{H} x}{\beta_{e}} \frac{\mathrm{d} p_{2}}{\mathrm{~d} t} \\
& Q_{H 1}-A_{H} \frac{\mathrm{d} x}{\mathrm{~d} t}=\frac{V_{1}+A_{H} x}{\beta_{e}} \frac{\mathrm{d} P_{1}}{\mathrm{~d} t}
\end{aligned}
$$

缓冲液压缸运动部件运动微分方程

$$
m_{H} \frac{\mathrm{d} x^{2}}{\mathrm{~d} t^{2}}+B_{v} \frac{\mathrm{d} x}{\mathrm{~d} t}+2 k_{t} x=\left(P_{1}-P_{2}\right) A_{H}
$$

式中, $V_{1}$ 为缓冲液压缸 $A(B)$ 腔初始容积; $m_{H}$ 为缓 冲液压缸运动部件质量; $k$ 为弹簧刚度。

同理，控制液压缸 2 的流量连续性方程为

$$
\begin{aligned}
& Q_{B 2}-2 A_{0} \frac{\mathrm{d} Y_{2}}{\mathrm{~d} t}=\frac{2 V_{0}+2 A_{0} Y_{2}}{\beta_{e}} \frac{\mathrm{d} P_{1}}{\mathrm{~d} t} \\
& 2 A_{0} \frac{\mathrm{d} Y_{2}}{\mathrm{~d} t}-Q_{A 2}=\frac{2 V_{0}-2 A_{0} Y_{2}}{\beta_{e}} \frac{\mathrm{d} p_{2}}{\mathrm{~d} t}
\end{aligned}
$$

式中, $Q_{B 2}$ 为 $B$ 腔出油流量; $Q_{A 2}$ 为 $A$ 腔进油流量。 单个控制液压缸运动部件运动微分方程如下

$$
m_{k} \frac{\mathrm{d}^{2} Y_{2}}{\mathrm{~d} t^{2}}=\left(P_{1}-P_{2}\right) A_{0}-B \frac{\mathrm{d} Y_{2}}{\mathrm{~d} t}-F_{2}
$$

旁通节流阀的流量由下式表达

$$
Q_{r}=C_{d} A_{2} \sqrt{\frac{2\left(P_{1}-P_{2}\right)}{\rho}}
$$

式中, $Q_{r}$ 为流过节流阀流量； $C_{d}$ 为节流口流量系 数; $\rho$ 为油液密度; $A_{2}$ 为旁通节流阀节流孔面积。 在节点 $M$ 与节点 $N$ 处, 得到流量连续性方程

$$
\begin{aligned}
& Q_{B 1}=Q_{r}+Q_{H 1}+Q_{B 2} \\
& Q_{A 1}=Q_{r}+Q_{H 2}+Q_{A 2}
\end{aligned}
$$

式(4) (15)即为研究防折弯功能数学理论模型。

\section{2 二系减振功能数学模型}

当车体相对于转向架发生横摆与摇头运动时, 会有油液经过缓冲阀门组流入或者流出液压缸, 起 到缓冲减振的作用。此时防折弯液压系统可同时起 到二系横向减振器和抗蛇行减振器的作用, 此即为 防折弯液压系统的二系减振功能。

为了研究防折弯系统对横向振动的抑制作用, 此 处仅考虑列车受到横向振动时的情况。此时一个模块 内的两个控制液压缸运动完全相同, 而不同车体模块 内的两个控制液压缸独立工作，不会发生油液交换。 此时可将研究目标简化为一个车体模块下的液压系 统, 图 5 即为液压系统二系减振功能简化模型。

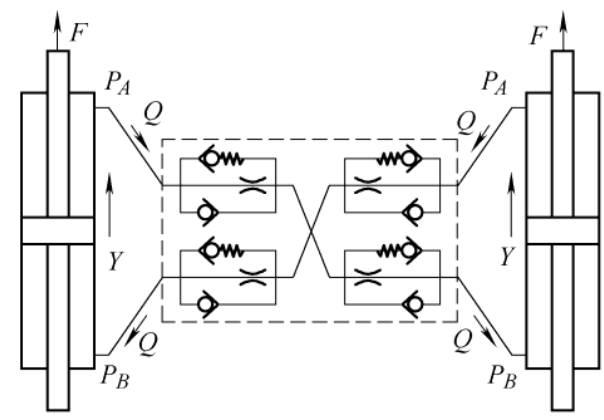

图 5 液压系统二系减振功能简化模型 
根据图 4 所示的简化模型, 假设控制液压缸受 力 $F$ 作用产生向上的位移 $Y, A$ 腔油液由于压力升 高而流出, $B$ 腔油液由于压力降低而流入, 针对液 压缸 $A$ 腔与 $B$ 腔, 根据流量连续性公式有

$$
\begin{aligned}
& A_{0} \frac{\mathrm{d} Y}{\mathrm{~d} t}-Q=\frac{V_{0}-A_{0} Y}{\beta_{e}} \frac{\mathrm{d} P_{A}}{\mathrm{~d} t} \\
& Q-A_{0} \frac{\mathrm{d} Y}{\mathrm{~d} t}=\frac{V_{0}+A_{0} Y}{\beta_{e}} \frac{\mathrm{d} P_{B}}{\mathrm{~d} t}
\end{aligned}
$$

液压缸运动部件微分方程

$$
m_{k} \frac{\mathrm{d}^{2} Y}{\mathrm{~d} t^{2}}=F-\left(P_{A}-P_{B}\right) A_{0}-B \frac{\mathrm{d} Y}{\mathrm{~d} t}
$$

流经节流阀和限压阀的流量为

$$
\begin{aligned}
& Q_{t}=C_{d} A_{1} \sqrt{\frac{2\left(P_{A}-P_{B}\right)}{\rho}} \\
& Q_{p}=C_{p}\left(P_{A}-P_{B}-\Delta P\right)
\end{aligned}
$$

式中, $C_{d}$ 为节流孔流量系数; $C_{p}$ 为限压阀流量系 数; $\Delta P$ 为限压阀开启压力。则

$$
Q=Q_{c}+Q_{p}
$$

式 $(16) \sim(21)$ 为二系减振功能数学模型 ${ }^{[15]}$ 。

\section{2 防折弯液压系统仿真分析}

\section{1 防折弯功能仿真分析}

根据第 1.1 节建立的防折弯功能数学模型, 在 Matlab/Simulink 中搭建仿真模型。

为了区别缓冲液压缸弹簧刚度和旁通节流阀直 径对回转刚度的影响, 设定如下运行模式。

第一阶段, $t=0 \sim 20 \mathrm{~s}$, 电磁阀 1 关闭, 电磁划 2 开启，此时只有缓冲液压缸接入系统。

第二阶段, $t>20 \mathrm{~s}$, 电磁划 1 和 2 均开启, 缓 冲液压缸和旁通节流阀均接入系统。

图 6 为分别改变缓冲油缸弹簧刚度和旁通节流 阀直径时防折弯系统回转刚度时间历程, 在第一阶 段(图 6a), 当系统中只有缓冲液压缸时, 回转刚度 为恒定值, 弹簧刚度越大, 回转刚度也越大。第二 阶段(图 6b), 当缓冲节流阀开启之后, 回转刚度随 时间增加不断减小, 且旁通节流阀直径越大, 回转 刚度减小越快。

\section{2 二系减振功能仿真分析}

根据第 1.2 节建立的二系减振功能数学模型, 在 Matlab/Simulink 中建立仿真模型, 施加正弦位移 信号, 获得减振系统的阻尼特性, 分析作用力 $F$ 与 活塞杆速度 $V$ 的关系(图 7)。

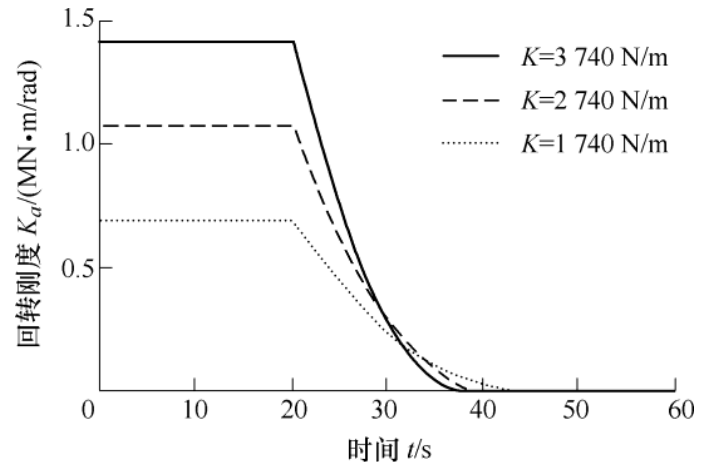

(a) 不同缓冲油缸弹簧刚度

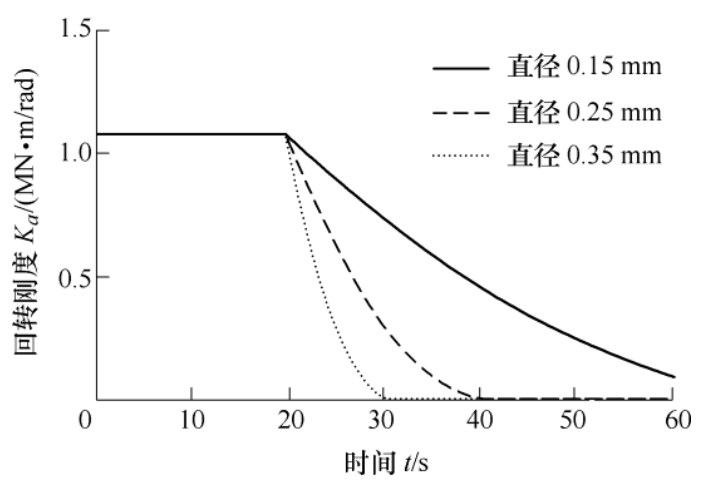

(b) 不同旁通节流阀直径

图 6 不同参数下防折弯系统回转刚度

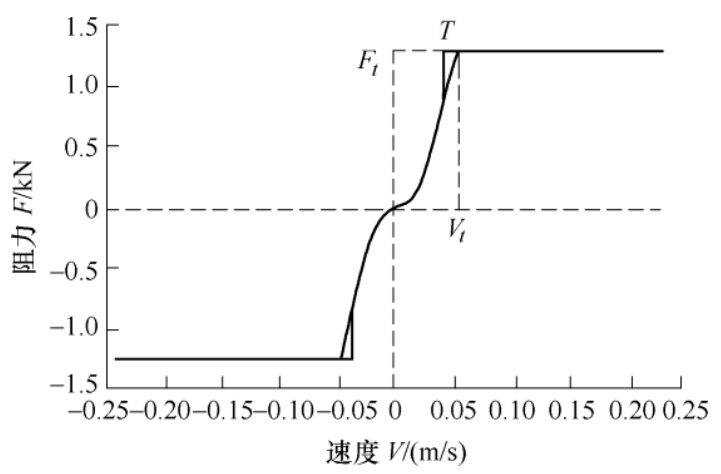

图 7 防折弯液压系统阻力特性

在阻力特性图中可以看到一个明显的转折点 $T, T$ 点所对应的速度值记为 $V_{t}$, 所对应的阻力值 记为 $F_{t}$ 。 当活塞速度小于 $V_{t}$ 的时候, 阻尼力随着 速度的增大而不断增大, 当活塞速度超过 $V_{t}$ 之后, 阻尼力基本保持不变。这是因为当活塞速度较小 时, 缓冲阀门组两端压差较小, 限压阀未打开, 仅有节流阀在工作；当活塞速度超过 $V_{t}$ 之后，缓 冲阀门组两端压差超过限压阀设定压力, 限压阀 打开，一部分油液经限压阀流过，因而压力基本 保持不变。

从阻力特性图中还可以发现 $F-V$ 曲线并不完全 重合, 这主要是由于系统建模时考虑了流体的压缩 性(式(16)、(17))。当不考虑流体压缩性时(图 8), 则 $F-V$ 曲线完全重合。 


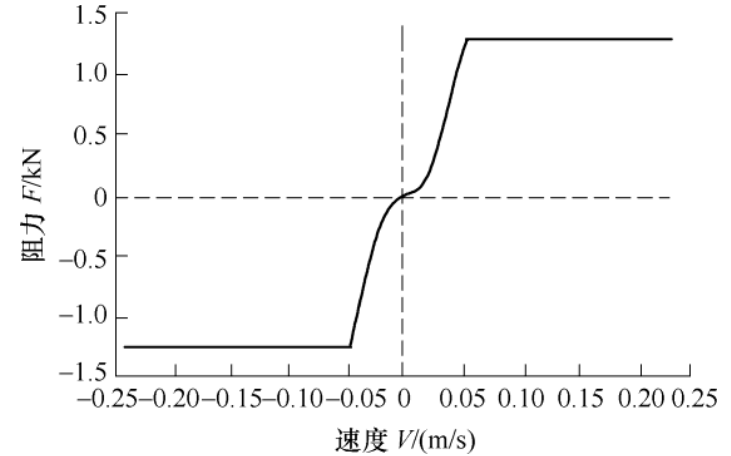

图 8 液压系统阻力特性(不考虑流体压缩性)

由防折弯系统原理分析可知，缓冲阀块节流孔 和限压阀对系统的阻尼特性起到决定性作用。为了 研究缓冲阀块中节流孔对系统阻力特性的影响, 设 置不同缓冲阀块节流孔直径和不同限压阀开启压 力，得到防折弯系统阻力特性图。

从图 9 可以看出, 不同节流孔直径下, 最大阻力 $F_{t}$ 基本保持不变, 且节流孔直径越大, 达到最大阻力 所对应的最小速度 $V t$ 也越大, 反映出随着缓冲阀节 流孔直径的增大, 防折弯系统等效阻尼系数逐渐减 小; 限压阀开启压力越大, 液压系统最大阻力 $F_{t}$ 也 越大, 而最大阻力对应的最小速度 $V_{t}$ 有小幅的增大。

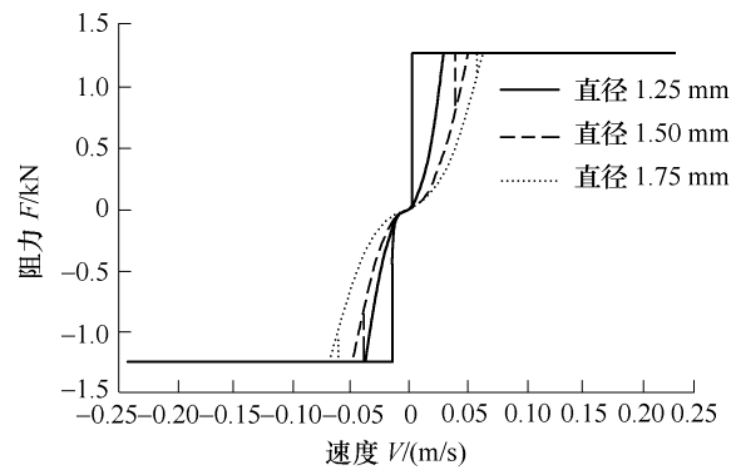

(a) 不同缓冲阀块节流孔直径

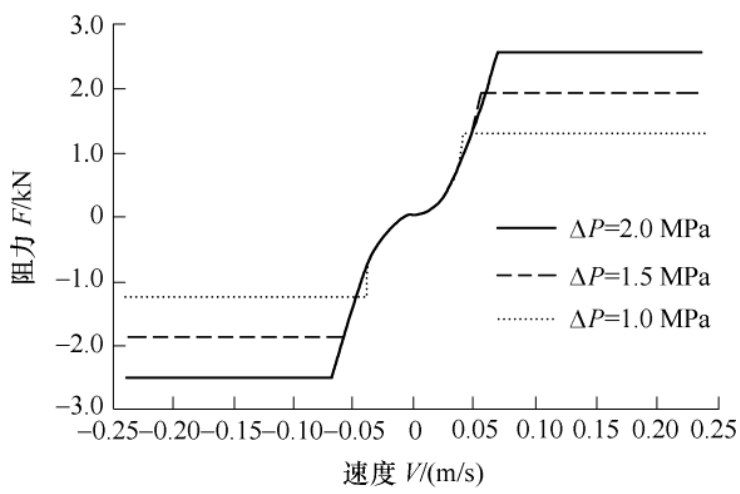

(b) 不同限压阀开启压力

图 9 不同参数下防折弯系统阻力特性

3 列车动力学-防折弯系统联合仿真

联合仿真模型包括列车动力学系统和防折弯液
压系统, 其中动力学系统通过 VI-Rail 软件搭建, 防 折弯液压系统通过 AMEsim 软件搭建。动力学系统 模型和液压系统模型搭建好之后再通过 Matlab 软 件下的 Simulink 工具箱进行联合仿真 ${ }^{[16]}$ 。联合仿真 过程中的数据交换为(图 10): 动力学模型产生车体 与转向架之间的活塞位移, 将该位移通过 Simulink 平台输入到液压系统中，从而使得液压系统产生相 应的活塞力, 该活塞力再通过 Simulink 反馈到动力 学系统中, 从而形成动力学系统与液压系统之间的 闭环数据交换。

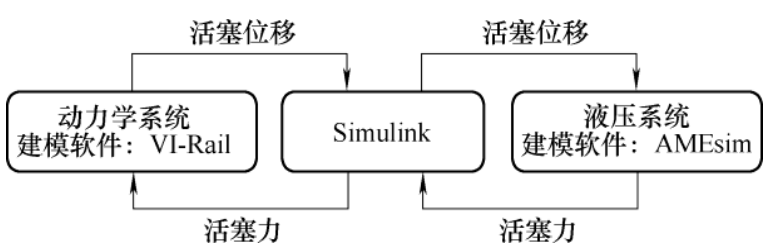

图 10 联合仿真各平台下数据交换

研究列车运行于小半径理想曲线时动力学性 能, 仿真曲线半径设定为 $25 \mathrm{~m}$, 曲线部分长为 10 $\mathrm{m}$, 无缓和曲线, 运行速度为 $11 \mathrm{~km} / \mathrm{h}$ 。图 11 是列 车通过曲线时, 四模块低地板有轨电车在有、无防 折弯系统下车体相对于转向架摇头角变化的时间 历程。

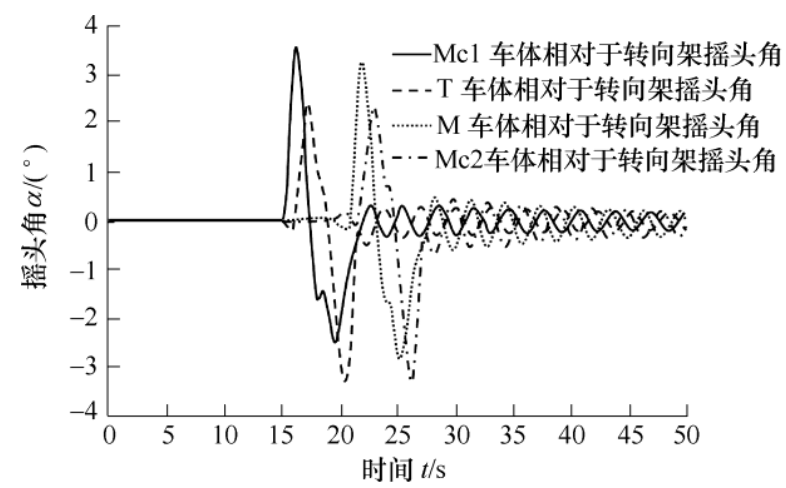

(a) 未加装防折弯系统

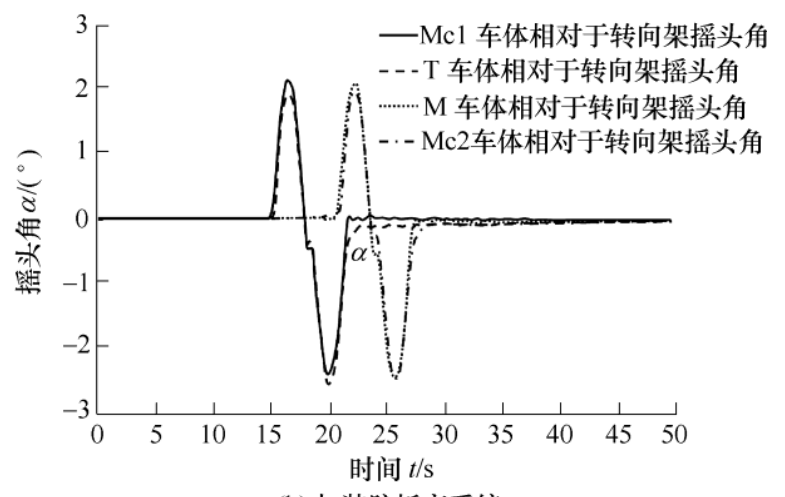

(b) 加装防折弯系统

图 11 车体相对于转向架摇头角时间历程

从图 11 中明显看出加装防折弯系统之后, 同一 液压系统所控制的两节车 $(\mathrm{Mc} 1$ 车与 $\mathrm{T}$ 车, $\mathrm{M}$ 车与 
$\mathrm{Mc} 2$ 车)车体相对于转向架摇头角曲线非常接近, 同 时车体相对于转向架的摇头角最大值也大幅减小。 当列车驶出曲线后, 无防折弯系统时(图 11a), 车体 相对于转向架摇头角存在小幅震荡现象, 这是由于 动力学模型二系阻尼较小原因, 当增加抗折弯系统 之后(11b), 列车出曲线后, 车体相对于转向架摇头 角便很快趋于稳定。

在 Mc1 车体前端外侧增加一限界测量点, 计 算车辆通过曲线时该点相对于轨道线路中心线 距离。

从图 12 中可以看到, 测点初始时刻相对于线路 中心线距离约为 $1.32 \mathrm{~m}$ 。无防折弯系统时, 最大距 离为 $1.66 \mathrm{~m}$, 偏移了 $0.34 \mathrm{~m}$; 加装抗折弯系统之后, 最大距离为 $1.52 \mathrm{~m}$, 偏移了 $0.20 \mathrm{~m}$ 。即加装抗折弯 系统前后, 测点相对于线路中心线距离偏移减小约 $41 \%$, 从而看出加装防折弯系统能有效减少列车通 过曲线时的限界问题。

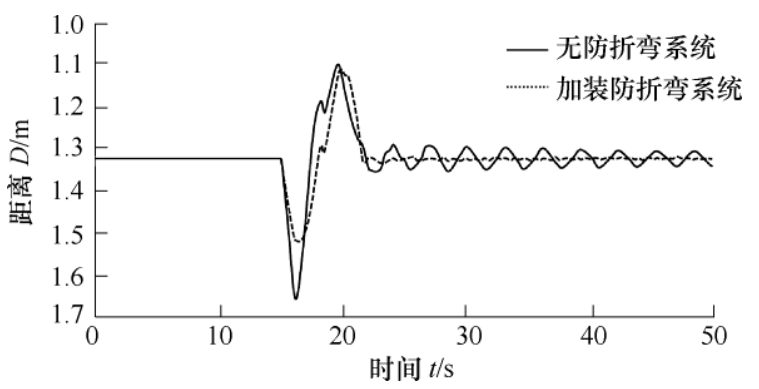

图 $12 \mathrm{Mc} 1$ 车体限界测点相对于轨道中心线距离时间历程

图 13 15 分别为有、无防折弯系统下, Mc1 车 各项动力学指标比较。从图 13 15 中可以看出, 加 装防折弯系统之后各项动力学指标最大值均小幅减

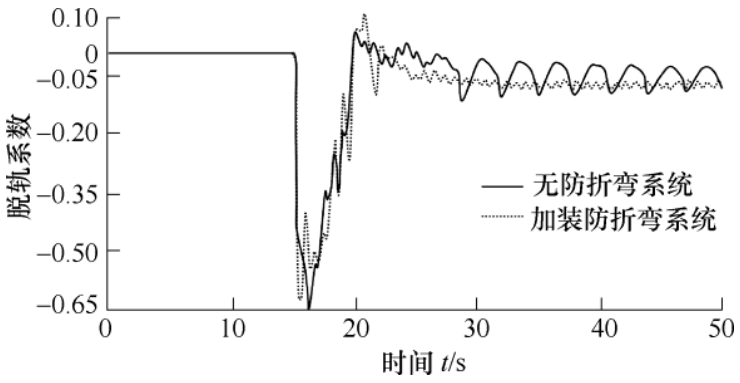

图 13 列车脱轨系数(曲线半径 $25 \mathrm{~m}$ )

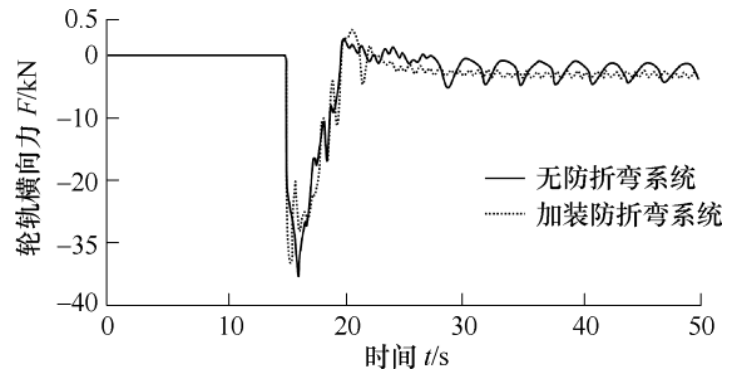

图 14 列车轮轨横向力 (曲线半径 $25 \mathrm{~m}$ )
小, 且列车运行出曲线后, 脱轨系数与轮轨横向力 趋于平稳。

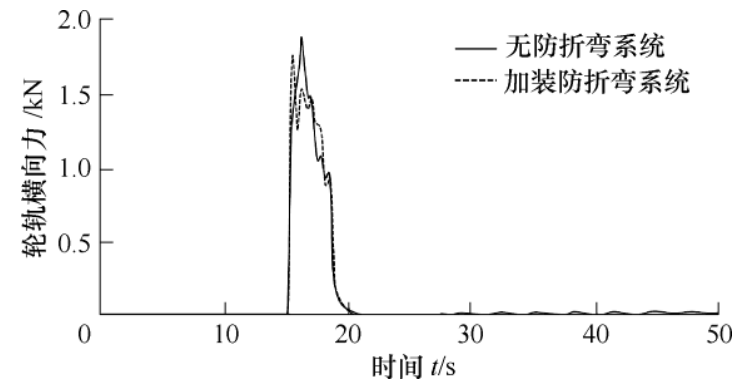

图 15 磨耗数(曲线半径 $25 \mathrm{~m}$ )

增大仿真曲线半径至 $70 \mathrm{~m}$, 曲线部分长 $153 \mathrm{~m}$, 存在过渡曲线, 列车运行速度为 $30 \mathrm{~km} / \mathrm{h}$ 。图 16 为 同一液压模块所控制的两节车 $(\mathrm{Mc} 1$ 车与 $\mathrm{T}$ 车)车体 相对于转向架摇头角时间历程, 图 17 为 $\mathrm{Mc1}$ 车转 向油缸反馈力时间历程。

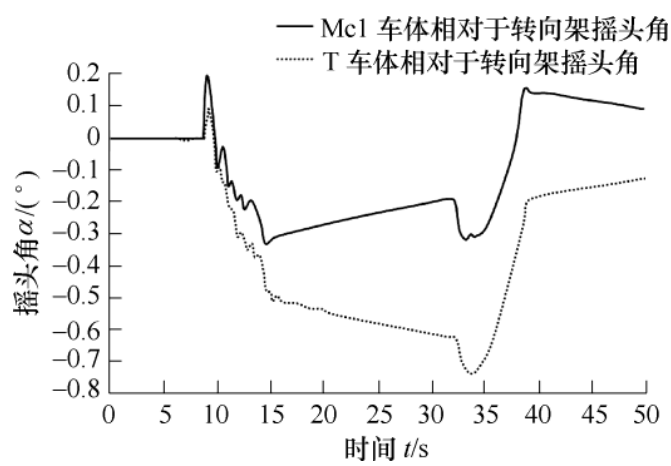

图 16 车体相对于转向架摇头角(曲线半径 $70 \mathrm{~m}$ )

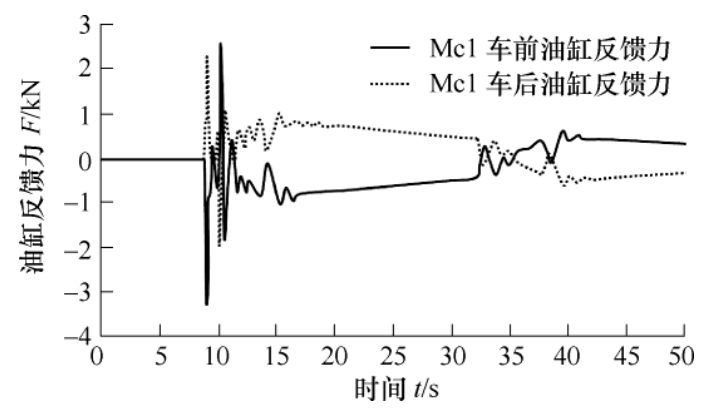

图 $17 \mathrm{Mc} 1$ 车前后转向油缸反馈力(曲线半径 $70 \mathrm{~m}$ )

从图 16 可以发现, 当曲线半径从 $25 \mathrm{~m}$ 增大 到 $70 \mathrm{~m}$ 之后, 防折弯系统能够使得列车在刚进入 曲线时，同一液压模块连接的两节车有较好的同 步性, 当列车完全进入曲线后, 同步性慢慢变差, 这主要由于旁通节流阀的开启，使得防折弯液压 系统的两条主回路相通, 通过两条主回路油液的 交流, 从而降低了防折弯系统的刚度, 这有利于 提高车辆运行平稳性。转向油缸作用力的峰值产 生于列车刚进入曲线的时刻(图 17), 最大值可达到 $3.4 \mathrm{kN}$, 当列车完全进入曲线后, 转向油缸反馈力 趋于稳定。 


\section{4 结论}

(1) 针对防折弯液压系统对于列车的不同约束 功能, 分别建立了防折弯功能数学模型和二系减振 功能数学模型, 分析了关键参数对防折弯系统的影 响。在此基础上通过防折弯液压系统-列车动力学系 统联合仿真, 研究了防折弯系统对列车系统作用原 理, 验证了联合仿真模型的正确性和可行性, 具有 建模精细和结果可靠的特点。

(2) 参数研究结果表明, 缓冲油缸弹簧刚度影 响同一液压模块内前后车体间辅助回转刚度大小, 旁通节流阀开启之后, 防折弯系统的刚度值会随着 时间增加而减小。缓冲阀块阻尼孔直径主要影响防 折弯系统等效阻尼, 而限压阀决定防折弯系统所能 提供的最大阻力。

(3) 加装防折弯系统后, 同一液压模块连接的 两节车 $(\mathrm{Mc} 1$ 车与 $\mathrm{T}$ 车, $\mathrm{M}$ 车与 $\mathrm{Mc} 2$ 车), 车体相对 于各自转向架绕 $Z$ 轴的摇头角有较好的同步性, 该 特性在小半径曲线上尤为明显; 车体相对转向架摇 头角显著减小，这能减小车辆通过曲线时车体横向 偏移量, 确保车辆通过曲线时在限界内。

\section{参 考 文 献}

[1] HERRERA V I, GAZTANAGA H, MILO A, et al. Optimal operation mode control and sizing of a battery-supercapacitor based tramway[C]// Vehicle Power and Propulsion Conference, Montreal, QC, Canada: IEEE, 2015: 1-6.

[2] SPAETH H , BECKER K P. Energy storage by capacitors[J]. European Transactions on Electrical power, 2002, 12(3): 211-216.

[3] HERRERA V, MILO A, CAZTANAGE L, et al. Adaptive energy management strategy and optimal sizing applied on a battery-supercapacitor based tramway[J]. Applied Energy, 2016, 169: 831-845.

[4] MITSUAKI H, YOSHIKI O. First 100\% domestic low-floor tram[J]. Mitsubishi Heavy Industries, Ltd. Technical Review, 2006, 43(1): 1-2.

[5] MUHAMMAD S. Low-floor LRVs move into second generation[J]. International Railway Journal, 1998, (1): 37-39.

[6] HARRY H. Three-stage programme put combino trams back on track[J]. Railway Grazette International, 2005, 161(10): 615-618
[7] 张徐. 四模块 100\%低地板有轨电车小曲线通过性能研 究[D]. 成都：西南交通大学, 2016.

ZHANG Xu. Study on small curve performance of four modules $100 \%$ low-floor trams[D]. Chengdu: Southwest Jiaotong University, 2016.

[8] 李创, 孙守光, 任尊松. 独立车轮转向架车辆曲线通过 性能分析 [J]. 北方交通大学学报，2003，27(5)：86-89.

LI Chuang, SUN Shouguang, REN Zunsong. Performance analysis of curve passing through on railway vehicle with independent wheels[J]. Journal of Northwest Jiaotong University, 2003, 27(5): 86-89.

[9] CZAUDERNA T, MANIOWSKI M. Stiffness analysis of 4 -link coupler mechanism used in low floor trams[J]. Acta Mechanica et Automatica, 2017, 11(2): 87-90.

[10] 姜宇飞, 谭敏明, 王力军. 储能式低地板有轨电车防折 弯系统分析 $[J]$. 技术与市场，2015，22(11)：26-31.

JIANG Yufei, TAN Minming, WANG Lijun. Anti-kink system of energy storage modern tram[J]. Technology and Market, 2015, 22(11): 26-31.

[11] 彭丽军, 谭敏明. 储能式现代有轨电车防折弯系统简介 [J]. 科技创新与应用, 2015(32): 149-150.

PENG Lijun, TAN Minming. Brief introduction of anti-kink system for energy storage modern tram[J]. Technology Innovation and Application, 2015(32) : 149-150.

[12] 王欢. $100 \%$ 低地板轻轨车辆结构型式与导向机理研究 [D]. 成都: 西南交通大学, 2008.

WANG Huan. Research of structure style and steering principle of $100 \%$ low floor light rail vehicle[D]. Chengdu: Southwest Jiaotong University, 2008.

[13] 黄江伟, 雷新红, 张登科, 等. 储能式有轨电车防折弯 系统试验研究 $[\mathrm{J}]$. 技术与市场, 2015，22(5): 26-32.

HUANG Jiangwei, LEI Xinhong, ZHANG Dengke. Experimental study on anti-kink system of energy storage modern tram[J]. Technology and Market, 2015, 22(5): 26-32.

[14] HUHTALA K. Modeling of hydrostatic transmissionssteady state, linear and nonlinear models[D]. Tampere: Acta Polytechnica Scandinavica Mechanical Engineering, 1996.

[15] 李晶, 朱先亮, 任利慧. 低地板轻轨车液压防折弯系统阻 尼特性分析[J]. 同济大学学报, 2017，45(7)：1044-1049. LI Jing, ZHU Xianliang, REN Lihui. Analysis of damping characteristics of hydraulic anti-kink system in low-floor trams[J]. Journal of Tongji University, 2017, 45(7): 1044-1049. 
[16] 李瑾, 邓卫华. AMESim 与 MATLAB/Simulink 联合仿 真技术及应用 $[\mathrm{J}]$. 情报指挥控制系统与仿真技术, 2004, 26(5): 61-64.

LI Jin, DENG Weihua. United simulation technique with AMESim and MATLAB/Simulink[J]. Information Command Control System \& Simulation Technology,
2004, 26(5): 61-64.

作者简介: 王小超, 男, 1992 年出生, 博士研究生。主要研究方向为车 辆动力学。

E-mail: wangxiaochao@tongji.edu.cn

陆正刚(通信作者), 男, 1966 年出生, 博士, 教授, 博士研究生导师。 主要研究方向为车辆动力学。

E-mail: luzhenggang@tongji.edu.cn 